\title{
Alignment and integration off-axis mirrors for space applications
}

Laure Tourrel, Christian Du Jeu, Patrick Robert, Alizee Loarer, Rupert Feckl, et al.

Laure Tourrel, Christian Du Jeu, Patrick Robert, Alizee Loarer, Rupert Feckl, Christoph Bartscher, Gaia Fusco, Thomas Deuschle, Kathrina Weiß, Didier Miras, Daniel Lamarre, Donny Aminou, Pascal Hallibert, "Alignment and integration off-axis mirrors for space applications," Proc. SPIE 11852, International Conference on Space Optics - ICSO 2020, 1185230 (11 June 2021); doi: 10.1117/12.2599462

SPIE Event: International Conference on Space Optics - ICSO 2021, 2021, Online Only 


\section{International Conference on Space Optics-ICSO 2020}

Virtual Conference

30 March-2 April 2021

Edited by Bruno Cugny, Zoran Sodnik, and Nikos Karafolas
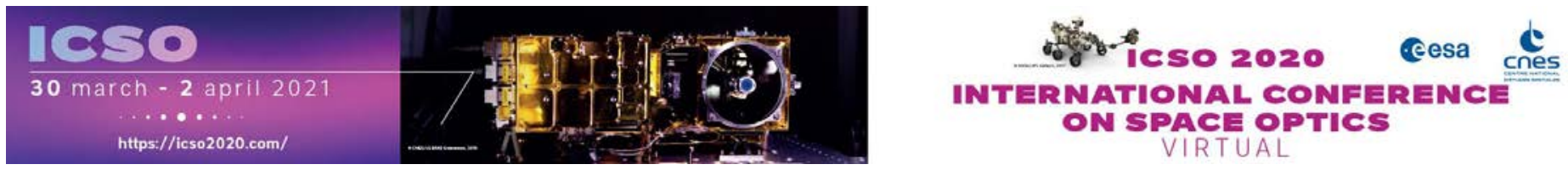

\section{Alignment and integration off-axis mirrors for space applications}

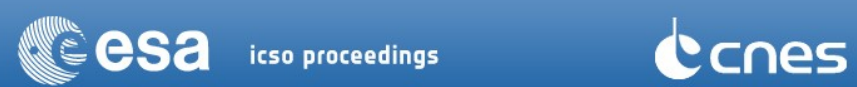




\title{
Alignment and inte gration off-axis mirrors for space applications
}

\author{
Laure Tourrel $^{1}$, Christian Du Jeu ${ }^{1}$, Patrick Robert ${ }^{1}{ }^{1}$ Alizée Loarer ${ }^{1}$ \\ Rupert Feckl ${ }^{2}$, Christoph Bartscher ${ }^{2}$, Gaia Fusco ${ }^{2}$, Thomas Deuschle ${ }^{2}$, Kathrina Wei $\beta^{2}$ \\ Didier Miras ${ }^{3}$ \\ Daniel Lamarre ${ }^{4}$, Donny Aminou ${ }^{4}$, Pascal Hallibert ${ }^{4}$ \\ ${ }^{1}$ Thales SESO, 530 rue Frédéric Joliot 13290 Aix-les-Milles, France \\ laure.tourrel/ christian.dujeu/patrick.robert/ alizee.loarer@fr.thales group.com \\ ${ }^{2}$ OHB System AG, Manfred-Fuchs-Str. 1, D-82234 Weßling - Germany \\ ${ }^{3}$ Thales Alenia Space, Allée des Gabians, 06156 Cannes Cedex - France \\ ${ }^{4}$ ESA, Keplerlaan 1, Nordwijk 2201 AZ, The Netherlands
}

\begin{abstract}
The field of earth observation requires increasingly complex optical instruments to meet the final requirements . The anticipation of instrument integration and alignment activities on the subsystem side is essential. Thales SESO manufactures opto-mechanical subsystems as sembled in such different space instruments.

The evolution of ins truments, as TMA type, concerning thereduction of the space allocated requires Thales SESO to offer opto-mechanical components and associated measurements that are increasingly precise and reliable.

The challenge here for Thales SESO is to manufacture, integrate and measure off-axis mirrors while ensuring accurate apexpositioning.

We will share here the results on the instrument of the MTG program for Telescope Optics subsystems of the two instruments FCI and IRS. Through a specific metrology scheme, including accurate scanning of the optical surface Thales SESO delivers to the customer a reliable and accurate location of the optical reference frame of each subass embly toward its mechanical reference frame.

From these relative location, the customer is able, in its assembly process, to "plug" the sub-assembly directly in its nominal position to start the alignment process with interferometric system.

The data transmitted by Thales SESO made it possible to anticipate each adjustment of the optical subsystems and to make a very accurate prediction of the alignment requirement. With the data measures by Thales SESO, our customers realize a very quick final alignment procedure, with minimum displacements, to meet the final goal.

In this process, the alignment budget is also minimized, leading to a final WFE largely under the predictions made by the customer before receiving the ass emblies.
\end{abstract}

Keywords: off-axis mirror, apexpositioning, accurate opto-mechanical design, integration mirror, alignment

\section{INTRODUCTION}

The MTG(Meteosat Third Generation) programis a collaboration with EUMETSAT andESA to performand innovate the next generation of meteorological satellite system.

The constellation is made up of sixs atellites MTG-I and MTG-S. Each has one of the two key instruments: on the one hand, the Flexible Combined Imager (FCI) and, on the other, the Infrared Sounder (IRS). 
The previous meteorological satellite, MSG is a satellite in constant rotation able to obtain:

- Full images with high resolution every $15 \mathrm{~min}$

- Detailed images (Europe) every 5 minutes

- A resolution of $1 \mathrm{~km}$ and $3 \mathrm{~km}$ depending on the spectral range.

As for MTG-FCI, which is a fixed satellite with a more complex thermal environment, it will be able to obtain:

- Full images with high resolution every 10 minutes

- $\quad$ Detailed images (Europe) every 2.5 minutes

- Higher resolution of imagery of 0.5-1 km and 1-2 km depending on the spectral range

- $\quad 16$ spectral ranges (grouped in 5 channels) instead of 12 (MSG)

One of the main challenges on the evolution between MSGand MTGis higher optical res olution of the instruments. The optical solution for these instruments is a TMA (Three mirrors Anastigmat) which include three off-axis mirrors in a small volume with a good optimization of main optical aberrations. Optical quality for each mirror in terms of precision and stability are the two criteria guaranteeing high performance of the instrument.

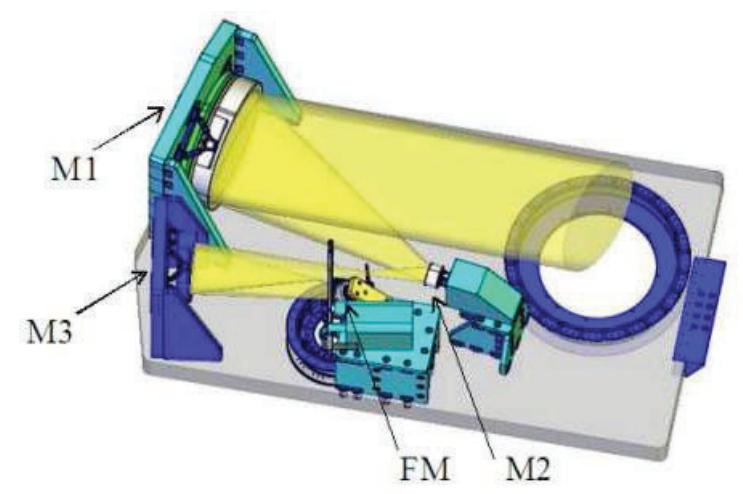

Figure 1-1: TMA optical path

Thales SESO participates in the development and manufacture of opto-mechanical components including thermal hardware on both FCI and IRS ins truments. Regarding mirrors, Thales SESO has designed, produced and tested all the FCI and IRS telescopes mirrors with eight different designs including six off-axis mirrors.

In terms of final telescope quality, the driver is to have mirrors perfectly aligned. The alignment is based on the teles cope WFEoptimization. As the three mirrors are off-axis, it is impossible to find the real optical axis of the as pheric surface during the alignment. Therefore a very accurate knowledge of virtual position of optical axis versus a real mechanical referential is needed. From the delivery of the equipped mirrors as manufactured at Thales SESO level, alignment of the telescope requires a good knowledge of the optical interface (namely the apex of the mirror for aspherical mirrors) toward its mechanical reference frame. Accuracy of this knowledge directly impacts :

- The physical adjustment range at telescope level

- The time and the convergence of the alignment process, inducing possibly many iteration loops (and therefore days of integration) before achieving the best WFE.

In this process, both theoptical reference frame and mechanical one have to be agreed together with the customer to have a process allowing the customer to reposition the mirror in its theoretical position before starting the alignment process.

In the frame of MTG FCI and IRS TO mirrors, the choice from the customer (OHB) was to build the mechanical reference frame of each sub-assembly from conical holes machined on the side of the mirrors ( 3 conical holes per mirror).

For other programs the choice is made of the mechanical reference frame in the interface plane: references on the mechanical parts (MFDs) interfacing the mirror as sembly to the telescope for example. 
The challenges are then:

- Design: Defining the proper reference frames in such a way to have an accurate measurement, taking into account the manufacturing/inspection steps

- Manufacturing: allowing to manufacture accurately both themechanical and the optical reference frames from their theoretical location

- Characterization: defining the metrology sequence/means/jigs allowing the accurate measurement of the defined reference frames (both optical and mechanical). Generally speaking, with modern measurement equipment mechanical reference frame is accurately located (in the range of less than $10 \mu \mathrm{m}$ ), whereas the optical reference frame needs to have a very precise knowledge of the optical surface of the mirror in the mechanical reference frame. This requires to have in the same measurement the location of the mechanical reference frame and a very accurate measurement of the optical surface which in general can be as low as a few hundreds of nm, depending on the exact sensibility of the mirror to misalignment.

- Integration: whenever the mechanical reference frame is not on the mirror itself but on the MFDs, mounting and dis mounting of the subassembly fromthe different supports through integration sequencerequires accurate repositioning.

Whatever the procedure it has to be applicable for small mirrors to very large one.

On MTG the procedure was applied for the different mirrors ranging from about $60 \mathrm{~mm}$ diameter to $400 \mathrm{~mm}$ diameter. Also Thales SESO succeeded to apply the same procedure on the TANGO demonstrator for Thales Alenia Space, with a diameter up to $1.5 \mathrm{~m}$.

\section{MANUFACTURING}

Thales SESO has a strong experience in the design and manufacture of complex opto-mechanical components ranging from large space mirrors (including Ø $1 \mathrm{~m} 50$ very lightweighted TANGO M1 zerodur mirror at $25 \mathrm{~kg} / \mathrm{m}^{2}$ and down to M2 MTG TO Ø $60 \mathrm{~mm}$ mirrors).

\subsection{Anticipate the manufacturing stages}

Upstreamof manufacture, the definition of the design must take into account many aspects related to the critical subjects of manufacture such as reference areas for measuring the component.

To guarantee the centering and orientation specifications of each component, we guarantee the consistency of the measurement reference system during the product life cycle.

Anticipating design changes during manufacturing is a key step in ensuring the centering specification (between 50 microns and a few hundreds of $\mu \mathrm{m}$ depending on the mirror sensitivity regarding the decentering) of the component's optical surface. The second point is to design and develop specific tools for the meas urement and integration of complex parts (To guarantee a fast and stable integration of the component before and during the measurement).

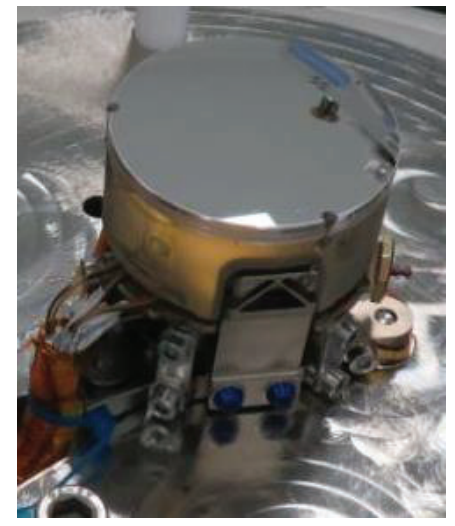

Figure 2-1: View on the difficulties of access to meas ure the mechanical reference frame after the final integration (M2 mirror FCI) 


\subsection{Component feasibility and meas urability difficulties}

Thales SESO designs, manufactures and integrates off axis mirrors of various sizes and designs. The required optical and opto-mechanical specifications require expertise and highly technical resources.

The main challenges of manufacturing each off axis mirror are:

- Polishing: The requirements on design and optical surface quality (see Table 2-1) require high technical expertise

- Measures and Integration :

- Measurements means of Thales SESO make it pos sible to respond to the need and to know with precision each location of the mechanical and optical reference frames.

- The final integration is complexand no longer allows access to customer repo sitories, which is why Thales SESO offers intermediate repositories to characterize each optical surface location in relation to mechanical interfaces.

Regarding the mirrors manufactured for FCI and IRS instruments, the component presenting the most important manufacturing challenges is as follows:

\begin{tabular}{|l|l|l|l|l|l|}
\hline $\begin{array}{l}\text { Name } \\
\text { Mirror }\end{array}$ & $\begin{array}{l}\text { Mechanicals } \\
\text { dimensions }\end{array}$ & $\begin{array}{l}\text { Shape of } \\
\text { Optical design }\end{array}$ & $\begin{array}{l}\text { Best fit } \\
\text { vers us spherical }\end{array}$ & $\begin{array}{l}\text { Polishing } \\
\text { requirements }\end{array}$ & $\begin{array}{l}\text { Manufacturing } \\
\text { difficulty criterion } \\
{[1][2]}\end{array}$ \\
\hline M1 FCI & $\varnothing 346 \mathrm{~mm}$ & Off axis concave & $640 \mu \mathrm{m}$ & $<33 \mathrm{~nm}$ RMS WFE & $0,82 \mathrm{~mm}$ \\
\hline
\end{tabular}

Table 2-1: Des cription of manufacturing requirements

\section{MEASUREMENTS}

Before carrying out a measurement campaign, Thales SESO makes sure to offer either a standard meas urement solution or, as here, to carry out a specific procedure. For this sequence we must carry out a characterization campaign of each final optical component surface

The sequence in place is the same for all mirrors, only the technical programming parameters obviously change depending on the size of the component and the measurement accuracy required.

\subsection{Definition of the meas urement means}

Thales SESO is equipped with thehigh-precis ion Leitzthree-dimensional machine equipped with a non-contact sensor that can measure flat or free-form optical surfaces.

This machine is able to take measurements of optical parts up to $1600 \mathrm{~mm}$ and guarantee measurement accuracy of $0.9 \mu \mathrm{m}+\mathrm{L} / 650 \mathrm{~mm}$.

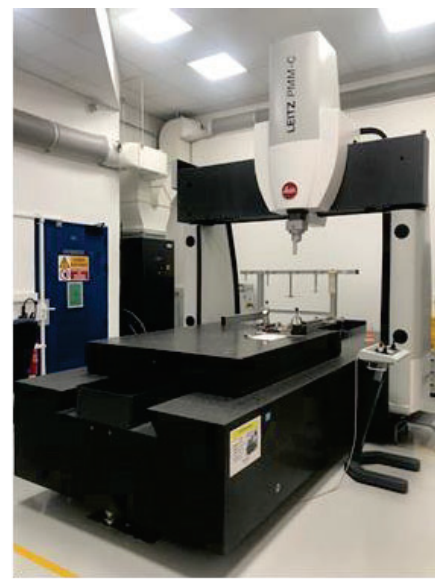

Figure 3-1: view of the PMM-C Leitz Machine 
Thales SESO is able to accurately link the location of the optical surface with the location of the mechanical referential. Firstly, by measuring each mechanical part with conventional ball probes (flatness and location interfaces) and by measuring each opticalsurface without contact @Precitec probe. This non-contact sensor makes it possible to scan opticalsurfaces at high speed without losing measurement precision. The @Precitec sensor reduces measurement uncertainties due to probing. It enables a large number of acquisitions to be acquired while guaranteeing good measurement repeatability

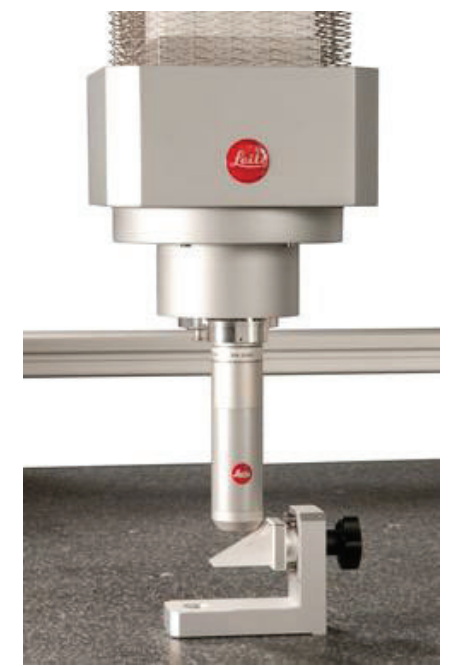

Figure 3-2: View of the @Precitec Sens or

\subsection{Meas urement characterization}

The measurement characterization campaign for each off-axis mirror is carried out before the completeintegration of the optical component into the mechanical assembly. The sequence is made to take into account the problem of the accessibility difficulties of the equipment once integrated.

The programming of each measurement is carried out using @QUINDOS software and guarantees homogeneity of the results for the same component by standardizing the metrology processes. Auto-run allows us to ensure fast and accurate measurement.

In addition, the repeatability of measurement is checked for each campaign by repeating the installation of the equipment and the measurement sequence 3 times.

\subsection{Accuracies of characterization meas ures}

Through a specific metrology scheme, including accurate scanning of the optical surface Thales SESO delivers to the customer a reliable and accurate location of the optical reference frame of each sub-as sembly toward its mechanical reference frame.

Thales SESO developed a dedicated software to build the tolerance budget of the inspection setup, depending on the mirror design. Accuracy includes using dedicated software to match the "as built" mirror (mechanical references vs optical axis) to the theoretical one.

From these relative location, the customer is able, in its assembly process, to "plug" the sub-assembly directly in its nominal position to start the alignment process.

With this approach and corresponding accuracy, the mirror in final use can be positioned on thetelescope with an optical surface centered to its theoretical location with a WFE residue deviating les s than a few hundred of nm from expectation. 
This was confirmed both on MTG mirrors (FCI and IRS), for which starting from a perfectly characterized mirror location, the alignment could start directly with interferometric measurement. Therefore it could go directly to the very accurate adjustment without need for preliminary alignment going through sophisticated procedure with intermediate steps and verifications.

For MTG, a combined effort (OHB and TSESO) was made to achieve a precise opto-mechanical characterization between as-built apex position and mechanical interfaces.

This procedure allowed OHB to reach for example a WFE of $330 \mathrm{~nm}$ rough from mounting on the overall telescope assembly for the first FCI telescope as mentioned in some mail reproduced below (mail form OHB 30/05/2018):

"I wanted to share some good news from Oberpfaffenhofen with you! The OHB AIT team has mounted the mirrors and the WFE is of the order of $330 \mathrm{~nm}$ (which is quite a good result for the first placement $(-)$ ). I take it as a proof that our efforts on the characterization were well invested, so my special thanks goes out to those who were working on this slightly tedious subject! “

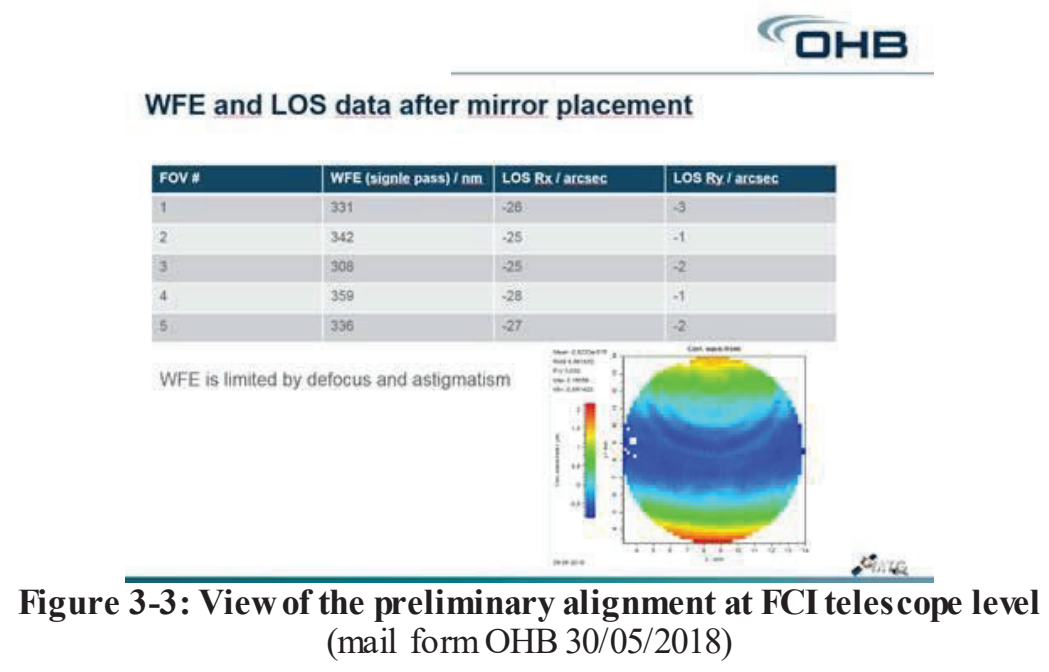

\section{RESULTS}

The results obtained by Thales SESO make it pos sible to correlate with great precis ion the location of the optical surface with the location of the mechanical reference frame. Given the good knowledge of Thales SESO measurements, for MTG FCI, starting with the preliminary alignment at $330 \mathrm{~nm}$ shown above, OHB was able to converge in one week (between May $30^{\text {th }} 2018$ and June $7^{\text {th }}$ 2018) to the final performance of $68 \mathrm{~nm}$ RMS whereas the target was in the range $100 \mathrm{~nm}$ at this step, including alignment residues (mail from OHB 08/06/2018). 


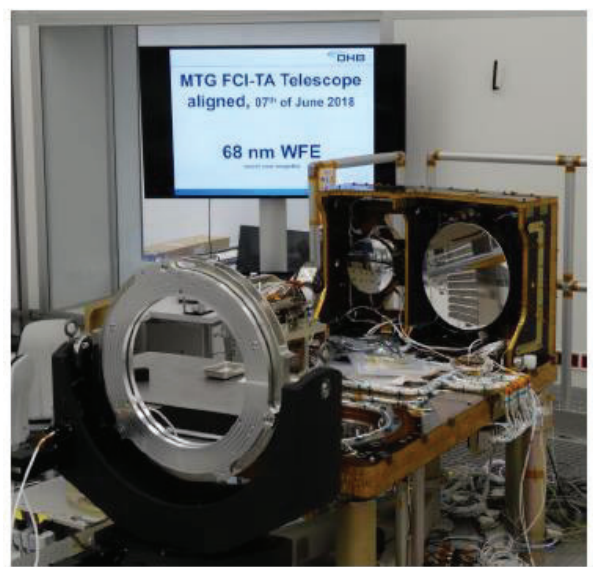

Figure 4-1: View of the final performance at FCI telescope level (mail from OHB 08/06/2018)

The same approach for TANGO demonstrator (1.5 m large telescope) leads to the following comment from Thales Alenia Space : The active telescope concept allows to simplify the assembly and integration phase. Several complex alignment ground support equipment are no more required. For instance, the telescope integration and alignment of the secondary mirror becomes one order of magnitude relaxed and is managed by theodolite means during mechanical integration thanks to the M2 5 Degree of Freedom mechanism. (Thales Alenia Space mail January 2021).

\section{CONCLUSION}

Alignment and integration off-axis mirrors are two complex works that require many iterative loops to ensure that each opto-mechanical component is properly measured and integrated to reply customer specifications. Our main results concern a good match between the measurements carried out at Thales SESO and the alignment prediction then the instrument integration loop at the customer.

\section{AKNOWLEDGEMENTS}

The work for the Meteosat Third Generation(MTG) Programme has been performed under contract from the European Space Agency (ESA) with OHB and Thales Alenia Space.

\section{REFERENCES}

[1] C. du Jeu, "Criterion to appreciate difficulties of aspherical polishing," Proc. SPIE5494, 113 (2004).

[2] Christian du Jeu, Julien Fourez, Has san El Handrioui, Matthieu Gilles, "Definitions of criteria for as sessing feasibility and measurability of freeformsurfaces," Proc. SPIE 11175, Optifab 2019, 1117510 (15 November 2019) 